\section{Traction-induced foveal damage predisposes eyes with pre-existing posterior vitreous detachment to idiopathic macular hole formation}

cause foveolar detachment and dehiscence. Improved ocular imaging technologies, however, revealed that perifoveal vitreous detachment is the vitreoretinal configuration associated with most early macular holes. ${ }^{2}$ High-resolution B-scan ultrasonography and optical coherence tomography (OCT) of patients with stages 1 and 2 idiopathic macular hole reveal a localized perifoveal vitreous detachment with foveolar vitreous adherence, strongly suggesting that perifoveal (stage 1) posterior vitreous detachment (PVD) is the primary pathogenic event in the idiopathic macular hole development. ${ }^{2}$

The observation that idiopathic macular hole occasionally develops in eyes with pre-existing spontaneous or surgical PVD has led some investigators to suggest alternative mechanisms of macular hole formation, such as primary degeneration of inner retinal layers at the central fovea. ${ }^{3}$ However, a more plausible explanation is that traction-induced foveal disruption occurring before or coincident with the vitreofoveal separation causes inner foveal damage with destabilization of the outer foveal layer. We describe two patients who had tractional damage to the fovea during PVD, which was followed sometime later by the formation of idiopathic macular hole. Sequential OCT imaging of these patients provided new insight into the mechanism of idiopathic macular hole formation in eyes with pre-existing PVD.

\section{Materials and methods}

Medical records of two patients were reviewed. Patients were examined clinically with fundus

\section{Introduction}

Idiopathic macular hole formation was initially hypothesized to be induced by tangential contraction of the attached prefoveal vitreous cortex. ${ }^{1}$ This tangential traction was proposed to

Keywords: macular hole; posterior vitreous detachment; vitreofoveal traction development of idiopathic macular hole in the setting of pre-existin

Methods Patients were examined clinically high-definition optical coherence tomography (OCT) was used to characterize the structural changes in the fovea following PVD. vitreofoveal separation and were found by disruption and irregularity of the foveal contour with no evidence of a full thickness macular hole. Sequential examination of these patients demonstrated delayed formation of Conclusion Traction-induced inner foveal damage occurring before or coincident with destabilizes the fovea and predisposes some Eye (2012) 26, 792-795; doi:10.1038/eye.2012.21;
\end{abstract}

Aim To propose a new mechanism for the 
contact lens biomicroscopy. Serial scans with highdefinition OCT were used to characterize the structural changes in the fovea following PVD (Cirrus HD-OCT software version 3.0.0.64; Carl Zeiss Meditec Inc., Dublin, CA, USA).

\section{Results}

\section{Case 1}

A 70-year-old man presented with decreased vision in the right eye for 4 days. Corrected visual acuity was 20/50. Biomicroscopy of the macular region showed no evidence of a full-thickness macular hole. OCT imaging showed vitreomacular separation (stage 2 PVD) with a small operculum suspended on the posterior hyaloid membrane overlying the fovea (Figure 1a). There was evidence of foveolar disruption with irregularity of the foveal contour as well as discontinuity of outer foveal layers. Careful review of the entire macular cube imaged by the Cirrus HD-OCT unit (Carl Zeiss Meditec Inc.) showed no full thickness macular defect. The patient reported a drop in vision 8 weeks later. The visual acuity measured $20 / 80$, and contact lens biomicroscopy and OCT imaging of the macula revealed a full-thickness macular hole with thickened edges and mild surrounding epiretinal membrane (Figure 1b).
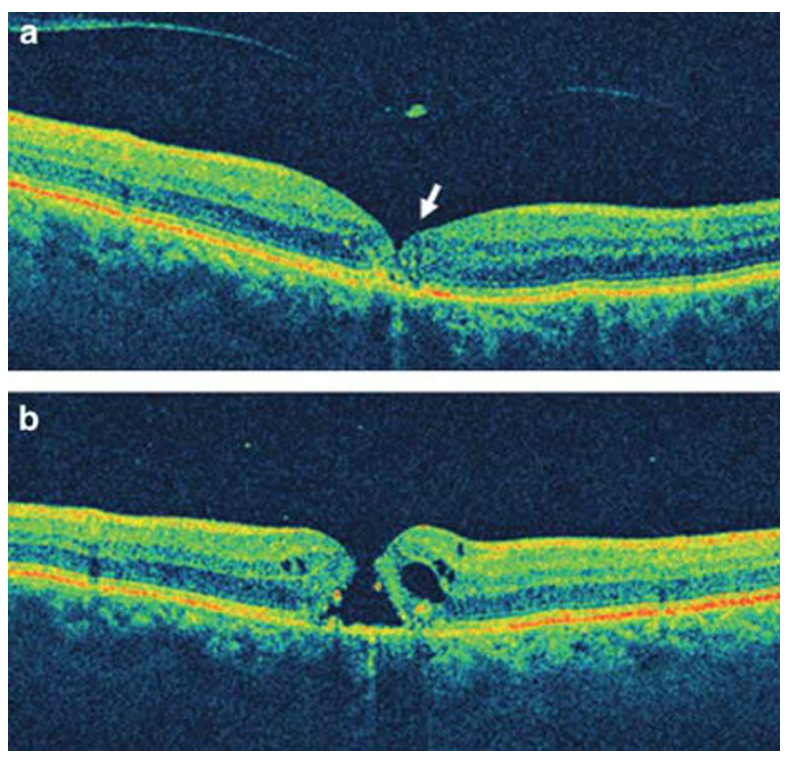

Figure 1 (a) Vertical OCT image of the right eye shows vitreous detachment over the macula with a small operculum. There is irregularity of the foveal contour and discontinuity of the outer foveal signals (arrow). (b) After 8 weeks, vertical OCT scan confirms the presence of a full-thickness macular hole with thickening of its edges.

\section{Case 2}

During evaluation of a full-thickness macular hole in the left eye, a 74-year-old man was found to have cystoid thickening of the fovea in the right eye by contact lens biomicroscopy. Corrected visual acuity in the right eye was $20 / 20$. OCT imaging of the right eye showed perifoveal vitreous detachment with traction-induced cystoid foveal thickening (Figure $2 \mathrm{a}$ ). On repeat evaluation of the right eye 3 months after the initial presentation, visual acuity measured 20/40 and the OCT demonstrated vitreofoveolar traction and cystoid thickening of the fovea with no evidence of macular dehiscence (Figure 2b). Eleven months after initial presentation, the visual acuity had improved to 20/30 and OCT showed complete vitreomacular separation with restoration of the foveal depression (Figures $2 c$ and d). However, careful examination of the OCT images revealed evidence for foveal disruption, including irregularity of the foveal contour and small cavitations in the inner foveal layers. The patient returned 4 months later with decreased (20/100) visual acuity. Biomicroscopy and OCT imaging of the macula showed a full-thickness macular hole surrounded by a mild epiretinal membrane (Figure 2e).

\section{Discussion}

In the last decade, high-resolution imaging of the vitreoretinal interface allowed identification of the near-universal association between stage 1 PVD and the early stages of idiopathic macular hole. ${ }^{2}$ This led to the hypothesis that anteroposterior and dynamic vitreofoveal traction caused by perifoveal PVD is the primary mechanism for idiopathic macular hole formation. This hypothesis was further supported by the findings of several investigators who demonstrated a close correlation between the extent of early macular hole and the progression of perifoveal PVD. ${ }^{4,5}$ Recently, Takahashi et $a l^{6}$ published multiple sequential OCT images of a stage 1A to stage 2 macular hole progression. This report elegantly illustrates the foveal split, outer layer disruption, and ultimately full-thickness dehiscence caused by traction from perifoveal PVD during the idiopathic macular hole development. It is now widely accepted that idiopathic macular hole is one of several macular disorders that represent complications of the early, slowly evolving stages of age-related PVD. ${ }^{2}$

The observation that a small minority of idiopathic macular holes develops in patients with well-documented pre-existing PVD has been difficult to explain and has led some investigators to propose alternative pathogenic mechanisms. Some have proposed that age-related 

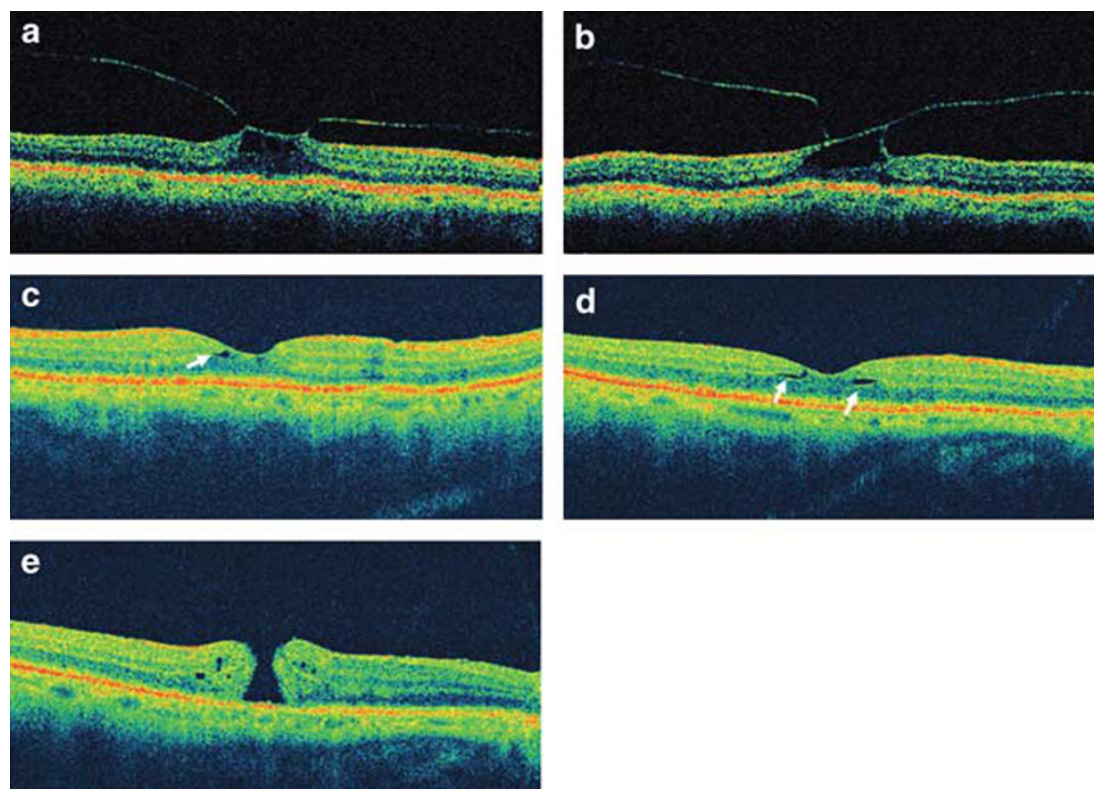

Figure 2 (a) Horizontal OCT image of the right eye at presentation, demonstrating stage 1 PVD with traction on the fovea causing cystoid foveal thickening. (b) Vertical OCT image of the same eye 3 months later, showing vitreofoveal traction with no evidence of a macular dehiscence. Eleven months after initial presentation, vertical (c) and horizontal (d) OCT scans show vitreomacular separation and restoration of the foveal depression. Signs of foveal damage include slight irregularity of the foveal contour and small cavitations in the inner foveal layers (arrows). (e) Horizontal OCT 4 months later demonstrates a full-thickness macular hole and mild epiretinal membrane.

degeneration of inner retinal layers at the central fovea is a primary event, predisposing some eyes to macular hole formation following tangential traction from epiretinal fibrocellular proliferation, which may function alone or in combination with vitreous traction. ${ }^{3}$ However, there is little evidence to support the concept of age-related degenerative changes in the inner fovea. In addition, ultrastructural analysis of the vitreomacular interface strongly suggests that the epiretinal fibrocellular proliferation often seen in association with idiopathic macular hole is a secondary rather than primary phenomenon. ${ }^{7}$

We propose that traction-induced foveal damage occurring before or coincident with either spontaneous or surgical vitreofoveal separation may destabilize the fovea, predisposing a minority of eyes to macular hole formation at a subsequent time. As in our patients, the foveal disruption may be subtle and therefore not initially cause significant vision loss or clinically apparent changes in foveal architecture. However, our cases suggest that if the tractional foveal damage results in sufficient compromise of structural integrity, fullthickness macular dehiscence may appear many weeks to months after vitreous separation.

We suggest at least two mechanisms by which vitreous traction on the inner aspect of the foveola could destabilize the outer foveal layer, rendering the macula susceptible to subsequent full-thickness dehiscence under relatively minor stress. First, because the Müller cell cone is believed to provide critical structural support for the fovea, its tractional separation from the photoreceptor and Henle fiber layers likely weakens lateral adhesion within the outer foveola. ${ }^{8}$ Second, as illustrated by Takahashi et al, ${ }^{6}$ disruption and even frank dehiscence of the outer foveal layer can occur when vitreous traction is transmitted to the photoreceptor layer through Müller cells.

Although vitreofoveal tractional damage may heal in most eyes and therefore go unnoticed, structural weakness may persist in a subset of eyes, rendering the fovea susceptible to fracture when subsequently acted upon by forces that would not harm a healthy macula. Such forces likely include tangential traction from mild epiretinal membrane, which was seen in both of our cases. In addition, the 'drawbridge' effect of retinal hydration may become operative in eyes with sufficient disruption of the inner foveal layers, resulting in progressive widening of the foveal break as the retinal edges swell. ${ }^{9}$ These forces, acting alone or in conjunction, could plausibly cause enough tangential traction to create a hole in a fovea damaged by previous vitreous traction. 


\section{Summary}

\section{What was known before}

- Perifoveal (stage 1) posterior vitreous detachment (PVD) is the primary pathogenic event in idiopathic macular hole development.

- The exact mechanism of idiopathic macular hole formation in eyes with pre-existing spontaneous or surgical PVD is still debated.

What this study adds

- High-definition OCT imaging demonstrates that traction-induced foveal disruption prior to or coincident with the vitreofoveal separation causes inner foveal damage.

- We propose that inner foveal damage destabilizes the outer foveal layer and this is the primary pathogenic event predisposing eyes with pre-existing PVD to idiopathic macular hole formation

\section{Conflict of interest}

The authors declare no conflict of interest.

\section{References}

1 Gass JD. Idiopathic senile macular hole. Its early stages and pathogenesis. Arch Ophthalmol 1988; 106: 629-639.
2 Johnson MW. Posterior vitreous detachment: evolution and complications of its early stages. Am J Ophthalmol 2010; 149: 371-382.

3 Smiddy WE. Macular hole formation without vitreofoveal traction. Arch Ophthalmol 2008; 126: 737-738.

4 Haouchine B, Massin P, Gaudric A. Foveal pseudocyst as the first step in macular hole formation: a prospective study by optical coherence tomography. Ophthalmology 2001; 108: $15-22$.

5 Ito $\mathrm{Y}$, Terasaki H, Suzuki T, Kojima T, Mori M, Ishikawa K et al. Mapping posterior vitreous detachment by optical coherence tomography in eyes with idiopathic macular hole. Am J Ophthalmol 2003; 135: 351-355.

6 Takahashi A, Nagaoka T, Ishiko S, Kameyama D, Yoshida A. Foveal anatomic changes in a progressing stage 1 macular hole documented by spectral-domain optical coherence tomography. Ophthalmology 2010; 117: 806-810.

7 Schumann RG, Schaumberger MM, Rohleder M, Haritoglou C, Kampik A, Gandorfer A. Ultrastructure of the vitreomacular interface in full-thickness idiopathic macular holes: a consecutive analysis of 100 cases. Am J Ophthalmol 2006; 141: 1112-1119.

8 Gass JD. Muller cell cone, an overlooked part of the anatomy of the fovea centralis: hypotheses concerning its role in the pathogenesis of macular hole and foveomacualr retinoschisis. Arch Ophthalmol 1999; 117: 821-823.

9 Tornambe PE. Macular hole genesis: the hydration theory. Retina 2003; 23: 421-424. 